\title{
Strickers Karl der Große: Stabreimende Wortpaare, insbesondere mit Rücksicht auf das Rolandslied
}

\section{Stricker's Karl der Große: Alliterating word pairs, with special consideration of the Early Middle High German Rolandslied}

\author{
John M. JEEP' (1)
}

'Dr., German, Russian, Asian and Middle Eastern Languages and Cultures, Miami University, Oxford, Ohio, USA

ORCID: J.M.J. 0000-0003-2456-9579

\section{Corresponding author:}

John M. JEEP,

German, Russian, Asian and Middle Eastern Languages and Cultures, Miami University, Oxford, USA

E-mail: jeepjm@miamioh.edu

Submitted: 08.09 .2020

Revision Requested: 07.11.2020

Last Revision Received: 07.11.2020

Accepted: 23.11 .2020

Citation: Jeep, J. M. (2020). Strickers Karl der Große: Stabreimende Wortpaare, insbesondere mit Rücksicht auf das Rolandslied. Alman Dili ve Edebiyatı Dergisi - Studien zur deutschen Sprache und Literatur, 44, 1-22.

https://doi.org/10.26650/sds|2020-0021

\begin{abstract}
DEUTSCH)
Nach Feststellung des Bestands an stabreimenden Wortpaaren im frühmittelhochdeutschen Rolandslied und dem Erscheinen der neuen kritischen Ausgabe von Strickers Karl der Große (Singer), ist es angebracht, nach den Wortpaaren in Singers Karl zu fragen, sie zu sammeln und zu analysieren und diese im Kontext und in Beziehung zu anderen frühdeutschen Werken zu beschreiben. Bislang ist das Althochdeutsche und das Frühmittelhochdeutsche erschlossen worden, mit Zusätzen aus dem Mittelhochdeutschen, vor allem in der hochklassischen Epoche. Auf eine Liste der Wortpaare chronologisch nach dem Erscheinen im Text folgt die alphabetisch geordnete Einzelanalyse, in der der Kontext erschlossen wird, Bezüge zum Rolandslied und gegebenenfalls zu der früheren Überlieferung der Wortpaare hergestellt werden, wobei der Gesamtbestand der stabreimenden Wortpaare in diesem Werk gewürdigt wird. Stabreim wird als rhetorisches Mittel nach germanischer Tradition aufgefasst; das heißt, dass nur betonte Silben miteinander staben. Vokale staben untereinander. Die Verbindungen $\langle$ sk->, $<$ sp-> und $<$ st- $>$ staben je nur miteinander. Die knapp 50 stabreimenden Wortpaare in Strickers Karl werden mit den 42 im Rolandslied bezeugten Paaren verglichen, wobei insbesondere Änderungen Beachtung verdienen. Neue Paare warden identifiziert, während bekannte Paare in ihrer Gebrauchstradition erschlossen werden. Es stellt sich heraus, dass maßgebliche Nachschlagewerke in vielen Fällen die Wortpaare nur unvollständig dokumentiert haben. Somit wird die Geschichte der stabreimenden Wortpaare im Frühdeutschen neugestaltet. Weitere Untersuchung werden die Rolle der stabreimenden Wortpaare in der Geschichte der deutschen Sprache und ihrer Literatur klarer warden lassen.
\end{abstract}

Schlüsselwörter: Mittelhochdeutsch, Rhetorik, Stricker, Stabreim/Alliteration, Karl der Große

\section{ABSTRACT (ENGLISH)}

Following the cataloging of the body of alliterating word pairs in the Early Middle High German Rolandslied (Song of Roland) and the appearance of a new critical edition of Stricker's Karl der Große (Charlemagne), it is appropriate to explore the alliterating word pairs in Stricker's Karl, to collect, analyze, and describe them in 
relation to other Early High German works. Existing research on Old and Early Middle High German is enhanced by use of additional data from the Classical Middle High German period. Following a chronological listing of the word pairs as they appear in Karl, the context is revealed in an alphabetical catalog of the pairs; connections to the Rolandslied and, when applicable, to the previous history of the word pairs, are revealed, thereby presenting an assessment of the nature of the catalog of the pairs in this work. Alliteration (stave rhyme) is understood here as following the Germanic tradition of only "staving" stressed syllables, while all vowels alliterate with other vowels. The initial combinations $\langle$ sk- $\rangle,\langle$ sp -$\rangle$, and <st-> stave only with themselves. The approximately 50 alliterating word pairs in Stricker's Karl are compared with the 42 pairs documented in the Rolandslied, with special attention given to any changes. New pairs are identified, while known ones are assessed within their tradition of usage. This exploration suggests that many standard reference works have only represented the history of alliterating word pairs in the early stages of German in an incomplete fashion. This study, therefore, revises the history of the alliterating word pair in the early stages of the German language. Further studies will expand the catalog and allow for a more accurate appraisal of the role of alliterating word pairs in the history of German language and literature.

Keywords: Middle High German, rhetoric, Stricker, alliteration, Karl der Große (Charlemagne)

\section{EXTENDED ABSTRACT}

Following the cataloging of the body of alliterating word pairs in the Early Middle High German Rolandslied (Song of Roland) and the appearance of a new critical edition of Stricker's Karl der Große (Charlemagne), it is appropriate to conduct an inquiry into the alliterating word pairs in Stricker's Karl, to collect and analyze those word pairs, and to describe them in relation to Karl's major source (the Rolandslied) and to the use of word pairs in other early German works. In the context of a long-term study, Old and Early Middle High German (circa 1180) have been studied exhaustively, using additional data from the Classical Middle High German period. This study builds upon a database of some 1,500 alliterating word pairs documented in the earlier stages of German. Stricker's Karl der Große, a reworking of the earlier Rolandslied, allows for comparison of the use of alliterating word pairs, whereby some are retained, others dropped, and still others reformulated. This offers an insight into the poetic inventory employed by Stricker. The known use of alliterating word pairs in his other works, albeit incomplete, is also included. A complete listing of all the word pairs he used should help enhance these findings. Following a chronological listing of the word pairs as they appear in the work; an alphabetical catalog of the pairs reveals their context, connections to the Rolandslied, and, when applicable, to the previous history of the word pairs. In this way, the nature of the catalog of the pairs in Stricker's work is assessed. The history of the alliterating word pairs reveals a combination of traditional usage and innovative rhetoric. The author navigates a wealth of options in formulating his tale, from everyday expressions to new pairings, and in consideration of rhyme constraints due to the nature of his work. Alliteration (stave rhyme) here is understood as following the Germanic tradition of only 
alliterating (or "staving") stressed syllables. This distinction can be critical. On the one hand, the Germanic poetic tradition used alliteration (in this strict sense) to form collocations that survived for centuries. On a more practical level, however, this matters for the history of an expression when a word evolves in relation to its initial phonemes, for example, an older "giloube" (which staves on ///), which ends up as /gloube/, hence alliterating with /g/. While other studies have included non-staving word pairs, we have elected to concentrate on this older tradition. In a similar fashion, one could also include triads, quadriads, or even longer chains, although their number is that much smaller and, hence, their overall impact less significant. In the Germanic tradition, all initial vowels alliterate with other vowels. The initial combinations $\langle$ sk- $>,\langle\mathrm{sp}-\rangle$, and $\langle$ st- $\rangle$ stave only with themselves. Within these parameters, the nearly 50 alliterating word pairs in Stricker's Karl are compared with the 42 pairs documented in the Rolandslied, with special attention paid to changes between the two. It should be noted that Stricker's Karl is approximately longer by one-third than the Rolandslied. Given the extant catalogs of early German word pairs, new pairs possibly coined by Stricker are identified, while known ones are assessed within the tradition of their usage. This study suggests that many standard reference works and the known secondary literature on Stricker's Karl der Große have only represented the history of alliterating word pairs in the early stages of German in an incomplete fashion. Therefore, this study provides a revision of the history of alliterating word pairs in the early stages of German. A complete listing of any rhetorical element is the foundation upon which an assessment of their functions can be attempted. Further studies will expand the catalog and allow a more accurate appraisal of the role of alliterating word pairs in the history of German language and literature. 


\section{Einleitung}

Nach Feststellung des Bestands an stabreimenden Wortpaaren im frühmittelhochdeutschen Rolandslied und dem Erscheinen der neuen kritischen Ausgabe von Strickers Karl der Große (Singer, 2016), ist es angebracht, nach den Wortpaaren in Singers Karl zu fragen und diese im Kontext und in Beziehung zu anderen frühdeutschen Werken zu beschreiben. Bartsch (1857, S. XCII) erwähnt den Stabreim in Wortpaaren in Strickers Karl, wobei er mit circa 25 Belegen nur auf knapp die Hälfte der stabreimenden Wortpaare gekommen ist („'magen und manne' 1010“ und "'gelt ode guot '2375“ sind als Fehleinträge zu deuten). Jensen (1885, S. 58f.) hat circa 20 identifiziert, wobei „hut und har" (S. 58) ebenfalls ein Fehleintrag darstellt. Schulze (1872-1873, S. 437) bezeichnet Stricker als formelreich. Jeeps Untersuchung (2016a) hat die Wortpaare im Rolandslied genannt, die auch in Strickers Karl belegt sind, für den das Rolandslied bekanntlich als Quelle diente (siehe Bastert, 2014; Ziegler, 2004, S. 81, Anm. 20; Geith,1994, S. 873f.; Kellett, 2008, S. 3 u. 51-56; Bumke, 125, S. 708; Haacke, 1959, S. 274-276; Singer, 2016, S. X). Kellett (2008, S. 158) findet die Beschreibung der Kampfszenen in Strickers Karl formelhafter als beispielsweise in Wolframs von Eschenbach Willehalm. So stand bislang eine vollständige Erfassung der stabreimenden Wortpaare in diesem und in den anderen Werken des Strickers aus. Verweise auf Handschriften von Strickers Karl im Folgenden beziehen sich auf Singers Verzeichnis (2016, S. XVI-XXV). Durchgehend wird Stabreim für Alliteration verwendet, da es sich hier um den germanischen Stabreim und nicht etwa auch den unbetonten Anlaut handelt. Gelegentlich wird auf die Chronik von Weihenstephan verwiesen, der Strickers Karl als wichtige Quelle diente (sieh Freitag, 1905).

Auf ein chronologisches Verzeichnis der Belege in dem Text (2.) folgt ein alphabetischer Katalog der Wortpaare (3.), eingeordnet nach den stabreimenden Elementen. Zitiert wird nach Singer (2016). Vokale mit übergeschriebenem $<$ e $>$ werden als Umlaute, $<\mathrm{u}>$ mit übergeschriebenem <o > wird als <uo $>$ widergegeben.

\section{Verzeichnis chronologisch im Text}

lip unde lebn

389

ir künege chroneten unde chürn

465

lip und leben

516

diu böste nnoch diu beste,

880 
baide ir lip vnd ir lant

liute unde lant.

brêchen und brennen

uof min ambt und uof min ê,

sin venjen und sin vasten,

mit gisel unde mit gebe.

lip und lant.

liut unde lant

her und hin,

lip unde lant.

di geisel und daz guot gar,

gisel [...] / und goldes

gisel und guot

ir und er

gisel und guot

wî si spilten und sprungen,

vriden und vristen

sig und sælde

daz ich uoze han oder inne

ein rehter und ein reîner,

durch den helm und durch die hirnschal

liute und lant

beide uozzen und innen

houbt und helm.

uozen und innen

houbt und helm

den schaden unt di schande,

durch den helm unt durh daz houbet.

diz fuogte got und ouch daz,

rein und reht.

die marter und di mennescheit

sælde und sige.

mit gewæfene und mit wenden,

her und hin,

beidiu verchiesen und verchlagen

den helm und den halsperch.
$1018(\mathrm{D}, \mathrm{F})$

1368

1590

1788

1818

2067

2643

2718

2828

2852

2898

$3234 f$.

3270

3288

3292

3626

3853

4240 (D)

4403

4722

4935

5186

5383

5739

5791

6030

6216

6391

6511

7484

8770

9267

9487

9925

10031

10113 


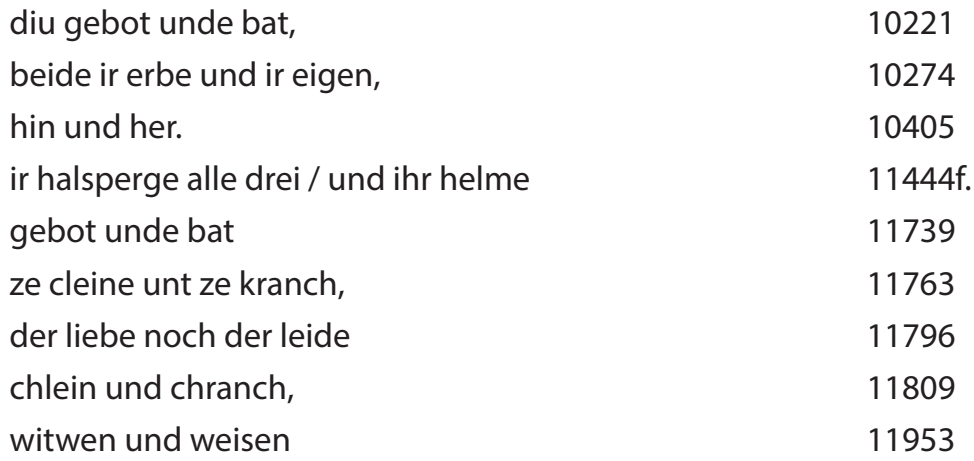

\section{Analyse der stabreimenden Wortpaare}

Die Analyse erfolgt alphabetisch nach dem ersten stabreimenden Glied; der <sch>Laut und $<\mathrm{sp}>$ erscheinen hinter $<\mathrm{s}>$.

uof min ambt und uof min ê,

1788

Bischoff Turpin beruft sich auf seine Position. Dieses Paar wird von einem weiteren allerdings nicht stabreimenden Paar gefolgt, in dem er sagt: „daz lere ich und rate." Soweit man sieht, ist dies der einzige Beleg für dieses Paar. Es steht nicht im Rolandslied, ist also als Ergänzung Strickers zu deuten. Jensen nennt den Beleg zusammen mit der folgenden Zeile $(1885$, S. 53) als Beispiel für Weitschweifigkeit. Zum Kontext siehe auch Freitag (1905, S. 115).

bat $>$ gebot

diu böste noch diu beste,

880

Hier beschreibt der Erzähler die besiegten Heiden in Spanien, die unfähig waren, Karl und seine Krieger zu besiegen. Schulze (1872-1873, 111) und Jensen (1885, 52 u. 58) verzeichnen diesen Beleg als stabreimend formelhaft. Dieses Paar steht nicht im Rolandslied, ist an dieser Stelle ein Zusatz des Strickers. Es darf als der früheste Beleg gelten.

gebot : bat (zwei Belege)

Das Paar ist bereits frühmittelhochdeutsch belegt (siehe Jeep, 2006, S. 23 u. 110), und oft danach (siehe Friedrich 2006, S. 119, s.v. ,biten', der das Paar als synonym bestimmt; 
ohne diese Belege). Jensen (1885, S. 54) führt zuerst nur einen Beleg, später (S. 58) dann beide. An einer Stelle in der Chronik von Weihenstephan findet man "des kaysers pot und pet" (sieh Freitag 1905, S. 158), für "rat" (10668) in Strickers Karl.

diu gebot unde bat,

10221

Pregmunda gibt Befehle, wie Karl an ihrem Hof empfangen werden solle. Die Chronik von Weihenstephan bietet an dieser Stelle „sprach“ (Freitag 1905, S. 162, Anm. 1).

gebot unde bat

11739

Kaiser Karl verordnet, so der Erzähler, was nach dem Urteil über Genilun geschehen soll.

brêchen und brennen

1590

Turpin rät, dass man die Gebetshäuser der Heiden zerstören soll. Dieses Paar ist im Rolandslied an dieser Stelle belegt (siehe Jeep, 2016a, S. 13). Jensen (1885, S. 58) bucht den Beleg.

diz fuogte got und ouch daz,

6511

Der Erzähler erklärt, was mit Abis im Zweikampf passiert ist. Hier bedeutet das Paar nicht etwa, dies und das', ,mancherlei' (so etwa Redewendungen, 2013, s.v.,dies'), sondern weist auf das, was gerade gesagt wurde und auf das, was unmittelbar folgt.

$$
\begin{aligned}
& \text { ê }>\text { ambt } \\
& \text { eigen }>\text { erbe } \\
& \text { er }>\text { ir }
\end{aligned}
$$

beide ir erbe und ir eigen,

10274

Der Erzähler berichtet, wie die neugetaufte Juliane (ehemals Pregmunda) ihren Besitz einer Stiftung übergibt. Diese Stelle ist eine Ergänzung zum Rolandslied, in dem lediglich „stiften" (8633) erwähnt wird. Dieses Wortpaar ist seit dem Althochdeutschen überliefert 
und seit Ende des 13. Jahrhunderts als Rechtsformel belegt (siehe Jeep, 2006, S. 25 u. 101; Schulze 1872-1873, S. 142f. mit diesem Beleg als Formel). Der Kontext zeigt, dass mit dem Paar Grundbesitz gegenüber fahrendem Gut (V. 10275) bezeichnet wird (DWB, 18541961, S. 3:96 u. 710, letzterer Eintrag mit Beleg). Die Reihenfolge der stabenden Glieder, die variiert, erklärt sich aus dem Reim. Die entsprechende Stelle in der Chronik von Weihenstephan (sieh Freitag 1905, S. 163, Anm. 4) ist ganz anders formuliert.

mit gisel unde mit gebe.

2067

Karl spricht hier über die Angebote Marsilies, durch Geisel und Tributzählung seinen guten Willen zu bekräftigen. Es entspricht dem Paar "mit gîselen unde mit gebe" im Rolandslied (V. 1514; dazu Jeep, 2016a, S. 14, mit der entsprechenden Literatur). Bartsch (1857, S. XCII) und Schulze (1872-1873, S. 146) führen diesen Beleg neben den drei anderen unten als Beispiel vom Stabreim mit "gisel". Jensen bucht diesen Beleg neben einem mit „guot" $(1885$, S. 58).

gisel sind her gesendet / und goldes

$3234 f$.

In diesem Satz berichtet Genilun Naymis gegenüber, was von den Heiden geschickt wurde. Auf die Geisel war mehrmals (dazu siehe hier oben und hier unten) in einem stabreimenden Wortpaar hingewiesen worden; jeweils variiert das zweite Glied. Zwei Handschriften bieten an dieser Stelle < die> vor < gisel> (Bartsch, 1857, S. 360). Das Paar scheint nur hier belegt zu sein, verbindet es vom Rolandslied "golt" (V. 2778) und "gisel" (2788) zu einem Wortpaar.

gisel : guot (drei Belege)

Bartsch (1857, S. XCII) und Schulze (1872-1873, S. 146) buchen alle drei Belege, Jensen $(1885$, S. 58) nur den ersten.

di geisel und daz guot gar, 2898

Genilun gibt Marsilies den Rat, Geisel und Geschenk an Karl zu senden. Diese Stelle weist auf den vorherigen Beleg, wobei „gebe" statt ebenfalls stabreimendem "guot" steht. Die Variante „geselle“ (Handschrift C, Singer 2016, App. zur Stelle) bringt Alliteration, aber keinen Stabreim mehr. 
gisel und guot

3270

Naymis berichtet Karl über die Gaben von Marsilies, auf die hier oben hingewiesen wurde.

gisel und guot

Naymis (wie im vorigen Beleg) spricht die Geisel und Geschenke an, die Karl zur Sicherheit von Genilun geboten wurden.

helm : halsperg (zwei Belege, einmal in umgekehrter Reihenfolge)

In der Kaiserchronik erscheinen drei stabreimenden Wortpaaren mit den Etyma "helm“, und zwar in Verbindung mit "halsperg" (siehe Jeep, 2006, S. 66 u. 103; auch Schulze, 18721873, S. 151), jeweils im Plural, einmal in umgekehrter Reihenfolge. Das Rolandslied hat einen Beleg (siehe Jeep, 2016a, S. 15), der aber nicht mit diesen Stellen zusammenhängt.

den helm und den halsperch.

10113

Hier schlägt Baligan auf Karl ein (siehe zum Kontext Kellett, 2008, S. 246).

ir halsperge alle drei / und ihr helme

$11444 f$.

Otto und seine zwei Begleiter binden ihre Rüstung an den geschlagenen Genilun. Die Angabe „alle drei" bezieht sich sowohl auf "halsperge“ als auf „helme“.

helm > houpt

durch den helm und durch die hirnschal

4935

Roland schlägt auf Alderot ein (zur Stelle siehe Kellett, 2008, S. 41 u. 221). Das Element „helm“ kann der Stricker an dieser Stelle direkt vom Rolandslied (4092) übernommen haben. Die Verbindung in einem Wortpaar scheint seine Prägung zu sein. Dies ist also der früheste deutsche Beleg für die Verbindung „helm : hirnschal“. Das Substantiv „,helm“ wird auch mit "houbt" und mit "halsperg" verbunden; dazu siehe hier unten. Laut Weber (2010, App.) gibt es auch die Variante mit Elision von „durch“ vor dem zweiten Substantiv. 
her: hin (drei Belege, einmal in umgekehrter Reihenfolge)

Alle Belege sind bei Schulze (1872-1873, S. 119) gebucht.

her und hin,

Ein noch heute geläufiges Wortpaar, wird es hier im selben Vers in Verbindung mit dem Verb "swuoren“ verwendet. Belegt ist das Paar seit dem Frühmittelhochdeutschen (Jeep, 2006, S. 104), siebenmal im Rolandslied, alle in der Reihenfolge "hin und her" der Adverbien. Die hier erstmals belegte Reihenfolge ist wohl durch den Reim bedingt. Das Paar greift inhaltlich auf, was im vorigen Vers chiastisch zum Ausdruck gebracht wird: „si swuoren im und swuor er in" (2827). Das Paar stellt eine Ergänzung zum Rolandslied dar.

her und hin, 9925

Baligan beschreibt sein Vorhaben, in der Schlacht in beiden Richtungen vorzudringen, um den christlichen Feind zu besiegen (zur Taktik und dieser Stelle gegenüber dem Rolandslied siehe Kellett, 2008, S. 148 u 153f.). Wie im vorigen Beleg ist die Reihenfolge durch den Reim bestimmt.

hin und her. 10405

Karl wiegt seinen toten Neffen Roland in seinen Armen. Die Reihenfolge der stabenden Adverbien ist durch den Reim („er" 10406) bedingt.

houbt : helm (drei Belege, einmal in umgekehrter Reihenfolge)

Bartsch (1857, S. XCII) verzeichnet die zwei ersten Stellen als Stabreim. Beide Belege sind mit dem Verb "spalten" und nur an diesen zwei Stellen im Werk verbunden (siehe Singer 2016, S. 434), und stehen im Reim mit "melm" (5738, 6029). Zingerle (1864, S. 128) führt nur einen Beleg. Es hat den Anschein (dazu hier unten), als habe der Stricker den Inhalt des Rolandsliedes zweimal in ein stabreimendes Wortpaar zusammengefügt, um es dann ein drittes Mal zu verwenden.

houbt und helm.

5739 
Der Erzähler berichtet, wie Engelîer Eschnermunt schlägt. Der Schlag bedeutet den Tod für Eschnermunt (5740), der auf den Sand „melm“ (6029) fällt. Schulze (1872-1873, S. 153) bucht diesen einen der drei Belege. Die Formulierung im Rolandslied, "den helm er uerscriet / ze tal durch sin houbit“ (4804f.) entspricht inhaltlich dem Paar hier.

houbt und helm

6030

Olivier erschlägt Margariez im Zweikampf (zur Stelle siehe Kellett, 2008, S. 232), wie im vorigen Paar im Reim mit „melm (6029). Wie im Paar hier oben entspricht die Formulierung im Rolandslied , "den helm er ihm erscalte. / daz houbet sich dar unter cluop“ (5094f.) dem Inhalt des Wortpaars. Schilter (1727, S. 35, Anm. II) hat schon auf diese Formulierung hingewiesen.

durch den helm unt durh daz houbet.

6391

Oliver erschlägt Chartan (zum Kontext siehe Kellett, 2008, S. 235). Hier bezeichnet das Paar den Tod Charans. Eine ähnlich entsprechende Formulierung wie in den zwei Paaren hier oben liegt nicht vor.

inne- > uoz-

ir und er

3288

Naymis erinnert Karl daran, dass dieser und Genilun ein Versprechen eingegangen sind. Die Verbform „gelobt“ passt zu beiden Pronomina im Nominativ. Pronomina werden oft in der Forschung zum stabreimenden Wortpaar nicht erfasst bzw. übersehen. Hier sei ein Nachtrag zum Bestand des „Armen Heinrich“ (siehe Jeep, 2018) geliefert: „nû sah er si an unde sich"' (Schwarz, 1967, S. 1231; zur Stelle siehe Schirokauer, 1957, S. 306).

beidiu verchiesen und verchlagen

10031

Baligan bietet Karl an, sich von seinen Klagen Karl gegenüber zu distanzieren. Ein nicht stabreimendes Wortpaar bildet das Objekt dieses Verbpaares: „min laster und min herceleit“ (10030). Baligan setzt das erste Verb später in derselben Rede wieder ein: „ich durch dich verchiesen will“ (10040). In seiner Ablehnung von Baligans Vorschlag greift Karl das erste Verb wieder auf: „, und verchiusest diniu apgot“ (10060). Eine direkte Vorlage 
im Rolandslied liegt nicht vor. Dieses Paar, sonst nicht belegt, scheint also vom Stricker zuerst formuliert zu sein.

clein(e) : kranch (zwei Belege)

Schulze $(1872-1873,115)$ bucht diese beiden Belege als formelhaft stabreimend.

ze cleine unt ze kranch,

11763

Herumstehende stellen vor dem Gerichtskampf fest, dass Pinabel ungeeignet sei, den Kampf zu gewinnen. Das Rolandslied hat an dieser Stelle lediglich „chranc” (8877). Dieses Paar scheint hier erstmals belegt zu sein.

chlein und chranch,

11809

Der Erzähler beschreibt Dietrich vor dem Gerichtskampf gegen Pinabel, wobei Bezug genommen wird auf die vorgenannte Stelle. Diese Beschreibung lässt Bartsch (1857, S. 343, Anm. zu Vers 1746 nach seiner Zählung) zweifeln, ob es sich hier um denselben Dietrich handeln kann, der früher (V. 1730 bei Singer, 2016) als stark beschrieben wurde. Das Rolandslied bietet hier keine Entsprechung. Man kann davon ausgehen, dass der Stricker das vorhin in ähnlicher Situation verwendete Paar hier wieder aufgreift.

ir künege chroneten unde chürn 465

Der Erzähler berichtet, dass fortan Karl als Kaiser in Aachen gekrönt und gewählt werden solle. Die von der Abfolge der Ereignisse eher verkehrte Reihenfolge der Verben lässt sich durch den Reim auf "verlürn" (466) erklären; ähnlich verhält es sich V. 3844: „beîde gechrönet und erwelt". Eine Variante <erchûrn> (Bartsch, 1857, V. 465, Hs. B) behält den Stabreim. Es liegt keine Entsprechung im Rolandslied für dieses Paar vor. Dies scheint der erste Beleg für dieses Paar zu sein. Der Stabreim mit „künege" (zur Graphie siehe Singer, 2016, S. XXXVI) verbindet das Syntagma zusätzlich.

$$
\begin{aligned}
& \text { lant }>\text { lip } \\
& \text { lant }>\text { liut(-) }
\end{aligned}
$$


leb- > lip

der liebe noch der leide

11796

Im Zweikampf zwischen Pinabel und Dietrich darf weder Hilfe noch Behinderung von außen angeboten werden. Das oft belegte Wortpaar (siehe Jeep, 2016b, S. 67f. mit weiterer Literatur; Zingerle, 1864, S. 143, bucht den Beleg) weist hier eine etwas ungewöhnliche Bedeutung auf; Jensen bestimmt sie als,keiner' (1885, S. 52; s. a. S. 58). Bartsch (1857, S. 429, Anm. zu Vers 11944) listet eine Variante in Handschrift D: <vnd > für $<$ noch $>$. Immer wieder variieren die Konjunktionen in stabreimenden Wortpaaren.

lip : lant (drei Belege, darunter eine Variante)

Das Paar erscheint nicht im Rolandslied. Jensen (1885, S. 58) bringt nur den zweiten dieser Belege.

baide ir lip vnd ir lant

$1018(D, F)$

Handschriften $\mathrm{D}$ und $\mathrm{F}$ liefern eine Variante zu einer Formulierung:,,ir lip, ir ere unde ir lant" (1018; siehe Bartsch, 1857, S. 334 und Weber, 2010, Anm. zum Vers), wobei die drei Substantive auf ein Wortpaar reduziert sind (zu Dreigliedrigkeit mit diesen Begriffen siehe auch Jensen, 1885, S. 56). Die Begriffe "lip" und "ere" (1014f.) werden nach einer Ansprache von Blanschandiez vom Erzähler an dieser Stelle wieder aufgegriffen. Im Rolandslied steht in der Rede von Blanschandiez unter anderem „liut unde lant" (V. 454, davor vom Erzähler V. 440; dazu Jeep, 2016a, S. 18).

lip und lant.

2643

Blandschandiez erzählt Marsilies, dass Karl ihm und seinen Männern ihren Besitz versichere.

lip unde lant.

2852

Hier verwendet der Erzähler biblische Vorstellungen (siehe Singer, 2016, Kommentar, 360; auch schon Jecklin, 1877, S. 163; Weinhold, 1895, S. 679, mit verunstaltetem Zitat „lip unde hant"), um Rolands Gegner zu verfluchen. Das Wortpaar ist ein Zusatz des Autors gegenüber der Quelle. 
lip : leb(e)n (zwei Belege)

Jensen $(1885,54)$ bestimmt das Paar als synonym bzw. verwandt und führt beide Belege.

lip unde lebn

Hier verspricht der Engel, dass Karl sein Leben in Spanien riskieren darf, ohne dass er es verlieren werde. Zum breiteren Kontext siehe Ziegler (2004, S. 27).

lip und leben

Der Erzähler berichtet, wie die zwölf Mitstreiter Karls ihn schützen werden. Eine Variante (Bartsch, 1857, Hs. F) liest <den leip und das leben>, ein Beispiel für ein Wortpaar mit den jeweiligen Artikeln. Bumke reduziert in seiner Übersetzung dieses Paares in einem anderen Werk des Strickers auf einen Substantiv "Leben“ (553f.), was auf die synonyme Eigenschaft der stabreimenden Glieder hindeutet.

liut(e) : lant (drei Belege)

Dieses Paar ist seit Althochdeutsch oft (Jeep, 2006, S. 106f.; 2016a, S. 18, mit weiteren frühmittelhochdeutschen Beispielen und Hinweisen auf Strickers Karl) mit beiden möglichen Reihenfolgen der stabreimenden Substantive belegt, hier wohl wegen der Reime in dieser Folge. Bartsch (1857, S. XCII) und Jensen (1885, S. 58) bringen jeweils nur zwei der drei Belege. Siehe van der Burg (1974, S. 94) zum Rolandslied V. 2580, wo Stricker das Paar nicht übernimmt. Überhaupt übernimmt der Stricker nur einen der vier „Rolandslied"-Belege (siehe Jeep, 2016a, S. 18f.). Im breiteren Kontext des Rolandslieds weist Ohly (1987, S. 96, zum Einfluss der Bibel auf das Rolandslied überhaupt S. 90f.) auf eine Stelle im Johannes-Evangelium hin, wo die Römer beschuldigt werden, „Land und Leute" (Joh. 11, 48) übernehmen zu wollen.

liute unde lant. 1368

Fürst Blanschandiez, der die Botschaft von Marsilies überbringt, stellt sich Karl vor. Das Paar stellt eine Ergänzung zum Rolandslied dar. 
Marsilies verspricht Genilun Macht und Besitz, falls der Plan gegen Roland gelinge. Auch für dieses Paar steht nichts Entsprechendes an dieser Stelle im Rolandslied.

liute und lant

5186

Oliver behauptet gegen Falsaron, seinen Besitz noch zu haben, solange Gott ihn schütze. Diesen einen Beleg hat der Stricker direkt aus dem Rolandslied (V. 4246, siehe Jeep, 2016a, S. 19).

die marter und di mennescheit

8770

Hier spricht Karl über die Bedeutung eines Traums, den er kurz zuvor gehabt hatte. Seine Kämpfer hätten die Mühen wissend und willentlich auf sich genommen. Dieses Paar, das keine Entsprechung im „Rolandslied“ hat, scheint nur hier belegt zu sein (siehe Schulze, 1872-1873, S. 161).

reht : rein (zwei Belege, einmal in umgekehrter Reihenfolge)

Bartsch (1857, S. XCII) bestimmt beide Stellen als Adjektive mit Stabreim. Das Paar scheint her zuerst belegt zu sein (siehe Schulze, 1872-1873, S. 116f. und Jensen, 1885, S. 58, die nur den ersten Beleg anführen).

ein rehter und ein reîner,

4722

Der Erzähler beschreibt Bischof Turpin als zuverlässigen Gesellen. Das Rolandslied hat an dieser Stelle: „sin herce was liuter und gâr“ (3901).

rein und reht. 7484

Oliver beschreibt an dieser Stelle Karl. Eine handschriftliche Variante (Bartsch, 1857, Hs. H) liest "gerecht", was den Stabreim behält. Das Rolandslied hat hier keine Entsprechung.

sig : sælde (zwei Belege, einmal in umgekehrter Reihenfolge) 
Die Verbindung der Etyma "sig" und "sælde" ist zweimal im Rolandslied sowie in anderen mittelhochdeutschen Werken belegt (dazu, mit weiterer Literatur Jeep, 2016a, S. 20).

sig und sælde

4240 (D)

Alderot grüßt Marsilies mit der Hoffnung, Mohamed möge ihnen zum Erfolg gegen die Christen helfen. Als Variante zu "sich und ere" bietet Handschrift D dieses Paar (siehe Singer, 2016, App. u. Weber, 2010, App.). Eine andere Handschrift (E) liest "salde und ere“ (zu diesem phraseologischen Wortpaar siehe Friedrich 2006, S. 342). Hier sieht man, dass die Begriffe,Sieg', 'Gnade' und,Ehre' manchmal austauschbar sind. Eine Entsprechung für dieses Paar bietet das Rolandslied an dieser Stelle nicht.

sælde und sige.

9267

Surian berät Baligan über die Gebete der Christen, dass ihr Gott ihnen Erfolg beschere. Bartsch (1857, S. XCII, fehlerhaft V. 9419 für 9417) notiert den Stabreim dieser Verbindung und den Bezug zum „Rolandslied“: „,sige unt ruom“ (V. 7977; siehe dazu Jeep, 2016a, S. 20; Hall, 1969, S. 355). Auch Jensen (1885, S. 58) bucht den Beleg. Die Reihenfolge kann man hier dem Reim zuschreiben.

den schaden unt di schande,

6216

Marsilies beklagt die negativen Erlebnisse, die ihm Karl gekostet habe. Dieses Paar ist seit dem Frühneuhochdeutschen (siehe Jeep, 2006, S. 112) und auch sonst bei Stricker (so Schulze, 1872-1873, S. 85f. \& Jensen, 1885, S. 58) überliefert. Das Rolandslied bietet keine Entsprechung an dieser Stelle.

Wî si spilten und sprungen,

3626

Hier träumt Karl, dass seine jungen Männer in Paris vergnügt Tätigkeiten ausüben, vor diesem Vers Singen, danach „sluogen unde stâchen" (3627), also ritterliche Übungen (zum Kontext siehe van Well, 2016, S. 106f.). Die gepaarten Verben lassen sich als solche durch die Metrik und den Stabreim bestimmen. Wegen Blattverlustes liegt keine Entsprechung im Rolandslied vor (Singer, 2016, Anm. zu V. 3622-3649). <sl> und <st> stellen im Deutschen keinen Stabreim dar, bis sich beide Anlaute zum Zischlaut <sch> 
entwickeln. Im „Alexanderlied“ findet man dieses Paar (siehe Jeep, 2011, S. 78, nachzutragen: DWB, 1854-1961, S. 16: 2326 mit dem früheren Beleg). Obwohl dieses Paar nicht im Rolandslied zu finden ist, mag es vielleicht in diesem Traum, der wegen Blattverlust nicht überliefert ist (siehe van Well, 2016, S. 106, Anm. 500), gestanden haben.

$$
\text { uoz(z)e(n) : inne(n) (drei Belege) }
$$

daz ich uoze han oder inne

4403

Für Singer (2016, S. 366, Komm. zur Stelle) ist dieser Vers nicht ganz klar, er schlägt vor: ,was ich habe an Besitz und Außenständen'. Für diese Stelle liegt keine Entsprechung im Rolandslied vor. Das Paar ,uz; in' ist sehr oft im Frühdeutschen belegt (siehe z. B. Jeep, 2006, S. 105 u. 114f), auch im Rolandslied (vier Belege, siehe Jeep, 2016a, S. 21-23, alle in dieser Reihenfolge). Jensen (1885, S. 50) bestimmt das Paar als adverbiell und idiomatisch für „überall“, bucht alle drei Belege.

beide uozzen und innen 5383

In der Schlacht wird die bedrohliche Hitze des Gefechts beschrieben. Dasselbe Paar wird an dieser Stelle im Rolandslied verwendet (dazu Jeep, 2016a, S. 22).

uozen und innen

5791

Der Erzähler beschreibt die Bewaffnung des Heeres von Hatte, wobei äußere und innere Stärken gemeint sind, wie auch im entsprechenden Beleg im Rolandslied (siehe Jeep, 2016a, S. 22; von der Burg, 1974, S. 310).

sin venjen und sin vasten,

1818

Vom Bischof Johan berichtet der Erzähler, dass jener Tag und Nacht seinen christlichen Pflichten nachkomme. Dieses erste von drei aufeinanderfolgenden Wortpaaren: „sin venjen und sin vasten, / sein wachen und sein gebet, daz er naht und tach tet" (18181820 ) ist das einzige von diesen dreien, das Stabreim aufweist. Die Verteilung auf die Verse, der Inhalt und der Stabreim unterstützen die Bestimmung als ein Wortpaar, und nicht etwa (nur) als eine Vierergruppe. Jensen (1885, S. 58) bestimmt das Paar als stabreimend idiomatisch. Ferner ist dieses Paar schon frühmittelhochdeutsch belegt, und 
zwar in der Kaiserchronik (siehe Jeep, 2006, S. 65 u. 102). Im Rolandslied heißt diese Stelle "mit venie joch mit vaste" (V. 1269, dazu Jeep, 2016a, S. 13). Springer beschreibt die zwei Verben an dieser Stelle in Karl als „büßerische und asketische Übung" (S. 356), ohne den Stabreim oder die Paarung zu nennen.

vriden und vristen

Roland erklärt Karl, er wolle den Heiden Frieden schaffen und sie schützen. Zur gleichen Formulierung im Rolandslied siehe Jeep (2016a, S. 13f.). Schulze (1872-1873, S. 102) führt nur diesen Beleg. Die Variante mit <und> ist durch zwei Handschriften gesichert. Die Formulierung als Akkusativ ergäbe auch einen Sinn: ,den Frieden sichern'. Singer (2016, S. 364) wiederholt (siehe Jeep, 2016a, S. 13f.) die Vermutung, es handele sich hier um eine Rechtsformel (ohne entsprechende Beweise). Das DWB verwendet ein stabreimendes Wortpaar, um das Verb "frieden“ zu erklären: „in schutz und schirm nehmen" (1854-1961, S. 4:188).

mit gewæfene und mit wenden,

9487

Der Erzähler beschreibt eine von Baligans aufgebotenen Scharen, wie sie sich auf den Kampf vorbereiten. Bartsch (1857, S. XCII) erkennt den substantivischen Stabreim. Es gibt im Rolandslied keine Entsprechung für diese Stelle. Das Paar scheint hier erstmals und vielleicht nur hier belegt zu sein. Zingerle (1864, S. 138) bringt den Beleg.

witwen und weisen

11953

Karls Ratgeber empfehlen, dass er Witwen und Waisen, für die ein christliches Oberhaupt Verantwortung trägt, Entschädigung leistet (siehe Singer, 2016, S. 431, s.v. „rihten“). Das Paar ist schon Alt- und Frühmittelhochdeutsch in der Regel in dieser Reihenfolge belegt (siehe Jeep, 2006, S. 69 u. 116f.). Wenn keine genaue Entsprechung für diese Stelle vorliegt, verbinden drei Belege im Rolandslied dieses Paar mit Kaiser Karl (siehe Jeep, 2016a, S. 24f.; zu V. 6868 nachzutragen ist Fischer, 1984, S. 167, Anm. 21).

\section{Ergebnisse und Aussichten}

Zum ersten Mal kann man genauere Aussagen über die Verwendung der stabreimenden Wortpaare im Rolandslied und in Strickers Karl machen. Die 49 hier in 
Strickers Karl (circa 12060 Verse) ermittelten stabreimenden Wortpaaren stehen 42 im Rolandslied (circa 9090 Verse) gegenüber (hier und zum Folgenden Jeep, 2016a, S. 27). Die Häufigkeit dieser Wortpaare in Strickers Version (1:246) liegt höher, wenn auch vergleichbar, als im Rolandslied (1: 216). Mehrfach im Karl überliefert sind: gisel : guot (drei Belege), her : hin (drei Belege), houbt : helm (drei Belege), lip : lant (drei Belege), liut(e) : lant (drei Belege), uoz(z)e(n) : inne(n) (drei Belege), gebot : bat (zwei Belege), helm : halsperg (zwei Belege), clein(e) : kranch (zwei Belege), lip : leb(e)n (zwei Belege), reht : rein (zwei Belege), sig : sæalde (zwei Belege), insgesamt also 30 Paare (mit 61\% fast identisch mit der Verteilung im Rolandslied: knapp 60\%), die mehrfach belegt sind. Es bleiben die 19 Paare, die in diesem Werk nur einmal überliefert sind. Davon sind folgende auch im Rolandslied belegt: brechen : brennen*, gisel : gebe*, halsberg : helm, in : ûz, venjen : vasten*, vriden : vristen*. Die mit * versehenen Paare sind, soweit man sieht, erstmalig im Rolandslied überliefert.

Neu in Strickers Karl (d.h. auch nicht im Rolandslied) sind, soweit man sieht: ambt : ê, böste : beste, gisel : goldes, helm : hirnschal, verchiesen : verchlagen, clein(e) : kranch, chroneten : chürn, marter : mennescheit, reht : rein, gewæfene : wenden.

Es zeigt sich, dass bei aller Übernahme in der Häufigkeit ähnlich bleibenden phraseologischen Inventars der Stricker neue stabreimende Wortpaare kreativ erdichtet, eine Art Tradition mit Innovation. Manche Paare bleiben sein Eigentum, andere werden vom Urheber wissend oder unwissend von anderen eingesetzt.

Manche Paare im Rolandslied findet man bei Stricker nicht: gold : gimmen, gote : goukelare, jaspis : jâchant, chrisolîte : calcedonie, lieb : lobehaft (dazu auch Schulze 18721873, S. 116), bisse : purpur, sagen : singen (dazu Heydler, 1840, S. IX, was in Jeep, 2016a, S. 19, nachzutragen ist; ebenfalls nachzutragen: Schwietering, 17, dann 22, mit einem Hinweis auf dieses Paar in Strickers Frauenehre), salmen : segene, ertunchen : ertwalen (dazu von der Burg, 1974, S. 26), unden : obne (dazu auch von der Burg, 1974, S. 94), vorloufte unde vederspil, witewen : waisen, wuofen : weinen (siehe auch van Well, 2016, S. 107f.; von der Burg, 1974, S. 27 u. 93; dafür steht in Karl 8554: „weinen und chlagen“), wuoft : weh.

Mit dieser Studie ist der Bestand an erfassten stabreimenden Wortpaaren des Mittelhochdeutschen erweitert, vor allem die Beziehung eines bedeutenden rhetorischen Mittels zwischen dem Rolandslied und Strickers Karl genauer beschrieben. Das stabreimende Wortpaar erweist sich immer wieder als dauerhaft und poetisch variabel. 
Der Bestand an stabreimenden Wortpaaren im übrigen Werk des Strickers zu erfassen, um so seine hier beschriebene Leistung zu ergänzen, bleibt Desiderat.

Weitere Wirkung mag die Verwendung von stabreimenden Wortpaaren aus dem Werk Strickers zu entdecken sein: so lauten Ergänzungen gegenüber Strickers Karl in der Weihenstephaner Chronik: „zuo sturmen oder zu strytten“ (siehe Freitag, 1905, S. 74), „priester und pischoff" (S. 97), (trotz sonstiger Kürzung hier, dazu siehe S. 112) „kain künig noch kai kayser" (S. 113); „edler künig und kayser" (S. 113).

Somit ist die Aufgabe, die stabreimenden Wortpaare des Deutschen historisch zu erfassen, ein Stück weitergekommen, wobei weitere Studien die hier gewonnenen Erkenntnisse vertiefen sollen. Es konnten auch Einsichten in die rhetorische Praxis des Rolandslieds und vor allem in Strickers Karl erörtert werden.

Begutachtung: Extern begutachtet.

Interessenkonflikt: Es besteht kein Interessenkonflikt.

Finanzielle Förderung: Dieser Beitrag wurde von keiner Institution finanziell unterstützt.

Peer-review: Externally peer-reviewed.

Conflict of Interest: The author has no conflict of interest to declare.

Grant Support: The author declared that this study has received no financial support.

\section{Literaturverzeichnis}

Bartsch, K. (Hg.) (1857). Karl der Grosse von dem Stricker. Bibliothek der gesammten deutschen National-Literatur von der ältesten bis auf die neuere Zeit 35. Quedlinburg / Leipzig: Basse.

Bastert, B. (2014). ,Karl der Große' von dem Stricker. In Historische und religiöse Erzählungen. (Hg.) Geert H. M. Claasenss / Fritz Peter Knapp / René Pérennec. Germania Litteraria Mediaevalis Francigena 4. Berlin / Boston: De Gruyter, 206-214.

Bumke, J. (1990). Höfische Kultur: Literatur und Gesellschaft im hohen Mittelalter. Bd. 1-2. 5. Aufl. München: dtv. Burg, U. v. d. (1974). Strickers Karl der Große als Bearbeitung des Rolandsliedes. Studien zu Form und Inhalt. Göppinger Arbeiten zur Germanistik 131. Dissertation Universität Bochum. Göppingen: Kümmerle.

DWB = Deutsches Wörterbuch von Jacob und Wilhelm Grimm. 16 Bände in 32 Teilbänden. Leipzig 1854-1961.

Quellenverzeichnis Leipzig: S. Hirzel, 1971. woerterbuchnetz.de

Fischer, M. C. (1984). The Idea of Christian Chivalry in the Chronicles of the Teutonic Order. Dissertation University of St. Andrews.

Freitag, O. (1905). Die sogenannte Chronik von Weihenstephan: Ein Beitrag zur Karlssage. Hermaea 1. Tübingen:

Max Niemeyer. 
Friedrich, J. (2006). Phraseologisches Wörterbuch des Mittelhochdeutschen: Redensarten, Sprichwörter und andere feste Wortverbindungen in Texten von 1050-1350. Germanistische Linguistik 264. Tübingen: Niemeyer.

Geith, K. E. (1994). Der Stricker. B. Werk. 1. Karl. In: Deutschsprachige Literatur des Mittelalters. Studienauswahl aus dem,Verfasserlexikon.' Berlin / New York: Walter de Gruyter, S. 871-875.

Haacke, D. (1959). Konrads Rolandslied und Strickers Karl der Große. In: Beiträge zur Geschichte der deutschen Sprache und Literatur 81, S. 274-294.

Hall, C. (1969). The Saelde-Group in Konrad's „Rolandslied“ and Stricker's „Karl der Große“. Monatshefte 61, 347360.

Heydler, W. F. (1840). Vergleichung des Rolandsliedes vom Pfaffen Conrad und des Karl vom Stricker [...]. Frankfurt a.O.: Trowitzsch.

Jeep, J. M. (2006). Alliterating Word-pairs in Early Middle High German. Phraseologie und Parömiologie 31. Baltmannsweiler: Hohengehren.

Jeep, J. M. (2011). The Straßbourg Alexander in the Context of the Early Medieval German Alliterating WordPairs. In: Amsterdamer Beiträge zur älteren Germanistik 68, S. 65-86.

Jeep, J. M. (2016a). Das Rolandslied: Stabreimende Wortpaare im Frühmittelhochdeutschen. Mediaevistik 29, 1138.

Jeep, J. M. (2016b). Handschrift W Gottfrieds von Strassburg und das stabreimende Wortpaar. In: Amsterdamer Beiträge zur älteren Germanistik 76, 31-88 und 208-269.

Jensen, L. (1885). Über den Stricker als Bîspel-Dichter, seine Sprache und seine Technik unter Berücksichtigung des "Karl" und "Amis". Dissertation Universität Marburg. Marburg: R. Friedrich.

Kellett, R. E. (2008). Single Combat and Warfare in German Literature of the High Middle Ages: Stricker's 'Karl der Große' und 'Daniel von dem Blühenden Tal'. London: Maney.

Ohly, F. (1987). Beiträge zum Rolandslied. In: Philologie als Kulturwissenschaft. Studien zur Literatur und Geschichte des Mittelalters. Festschrift für Karl Stackmann zum 65. Geburtstag. Ludger Grenzmann \& Hubert Herkommer \& Dieter Wuttke (Hg.). 90-135, Göttingen: Vandenhoeck \& Ruprecht.

Redewendungen $=$ Redewendungen und sprichwörtliche Redensarten. Idiomatisches Wörterbuch der deutschen Sprache. Duden 11. (2013). Dudenredaktion (Bearb.). 4. Auflage. Mannheim et al.: Dudenverlag.

Das Rolandslied des Pfaffen Konrad. Mittelhochdeutscher Text und Übertragung. (1970). (Hg.) Dieter Karschoke, Fischer Bücherei Bücher des Wissens 6004. Frankfurt am Main: Fischer.

Schilterus, J. (Hg.) (1727). Anonymi fragmentum de bello Caroli M. Contra Saracenos [...]. Ulm: Daniel Bartholomäus. Schirokauer, A. (1957). Gesammelte Studien Ausgewählt und eingeleitet von Fritz Strich. Hamburg: Ernst Hauswedell.

Schulze, C. (1872-1873). Die sprichwörtlichen Formeln der deutschen Sprache, I. Archiv für das Studium der neueren Sprachen und Literaturen 48, S. 435-450.

Schulze, C. (1872-1873). Die sprichwörtlichen Formeln der deutschen Sprache, II. Archiv für das Studium der neueren Sprachen und Literaturen, 49, S. 139-162. 
Schulze, C. (1872-1873). Die sprichwörtlichen Formeln der deutschen Sprache, III. Archiv für das Studium der neueren Sprachen und Literaturen, 50, S. 83-122.

Schwarz, E. (1967). Hartmann von Aue. Gregorius. Der arme Heinrich. Text. Nacherzählungen. Worterklärungen. ${ }^{2}$ 1986. Darmstadt: Wissenschaftliche Buchgesellschaft.

Schwietering, J. (1908). Singen und Sagen. Dissertation Universität Göttingen. Göttingen: E.A. Huth.

Singer, J. (Hg.) (2016). Strickers Karl der Große. In: Deutsche Texte des Mittelalters 96. Berlin / Boston: De Gruyter. Akademie Forschung.

Von Jecklin, C. (1877). Zu des Strickers Karl. Germania 22, 129-166.

Weber, S. (2010). Strickers „Karl der Große". Analyse der Überlieferungsgeschichte und Edition des Textes auf Grundlage von C., Schriften zur Mediävistik 18. Hamburg: Dr. Kovač.

Weinhold, K. (1895). Die altdeutschen Verfluchungsformeln. In: Sitzungsberichte der Königlich Preußischen Akademie der Wissenschaften zu Berlin. Zweiter Halbband. 667-703. Berlin: Verlag der Königlichen Akademie der Wissenschaften

Well, B. v. (2016). „Mir troumt hînaht ein troum“: Untersuchung zur Erzäh/weise von Träumen in mittelhochdeutscher Epik. Mit einer Abbildung. Dissertation Freie Universität Berlin 2015. Göttingen: V\&R unipress.

Ziegler, V. L. (2004). Trial by Fire and Battle in Medieval German Literature. Studies in German literature, linguistics, and culture. Rochester: Camden House.

Zingerle, I. V. (1864). Die Alliteration bei mittelhochdeutschen Dichtern. In: Sitzungsberichte der kaiserlichen Akademie der Wissenschaften zu Wien. Philosophisch-historische Classe, 47(1), S. 103-174. 